\title{
Casimir-Based Control Beyond the Dissipation Obstacle
}

\author{
Johan Koopman and Dimitri Jeltsema \\ Delft Institute of Applied Mathematics, Delft University of Technology, \\ Mekelweg 4, 2628 CD Delft, The Netherlands
}

\begin{abstract}
A prevailing trend in the stabilization of port-Hamiltonian systems is the assumption that the plant and the controller are both passive. In the standard approach of control by interconnection based on the generation of Casimir functions, this assumption leads to the dissipation obstacle, which essentially means that dissipation is admissible only on the coordinates of the closed-loop Hamiltonian that do not require shaping and thus severely restricts the scope of applications. In this contribution, we show that we can easily go beyond the dissipation obstacle by allowing the controller to have a negative semi-definite resistive structure, while guaranteeing stability of both the closed-loop and the controller.
\end{abstract}

Keywords: Passivity-based control, control by interconnection, Casimirs, dissipation obstacle.

\section{INTRODUCTION}

In the past decade, passivity-based control (PBC) has emerged as a control design method that respects, and successfully exploits, the physical structure of a system. Using the port-Hamiltonian $(\mathrm{pH})$ formalism, state-space control design methods are proposed that lead to con'trollers and subsequent closed-loop systems that admit a physical interpretation. In this approach the Hamiltonian ' (i.e., the internal stored energy) of the system is the focal point of the design method, serving as a Lyapunov function for stability and as a storage function for passivity; see (Schaft, 2000) for a basic introduction and (Duindam, 2009) for a comprehensive summary of the developments of the $\mathrm{pH}$ framework over the past decade.

Starting with the so-called energy shaping (ES) routine (Ortega, 2001), in which the closed-loop energy is shaped using static state feedback, numerous extension have led to a variety of control methods that set out to shape the system's energy, interconnection and dissipation structure. However, only the static full-state feedback methods are developed to a form that can be considered generic. Indeed, the interconnection and dampingassignment passivitybased control (IDA-PBC) method is shown (Ortega, 2008) to generate all stabilizing static state feedback controllers for $\mathrm{pH}$-systems.

Existing dynamic output feedback PBC strategies - the so-called control-by-interconnection (CbI) methods - are centered around the notion of Casimir functions, which statically relate the states of the controller to those of the plant (Schaft, 2000; Ortega, 2008). Although these Casimir-based control design methods are in some ways very attractive, they are not yet developed into a generic form. First of all, the input-output structure of both plant and controller is assumed to be power-conjugate, i.e., the input and output of both plant and controller is assumed to constitute a power-port and are thus of equal dimension. This can be seen as a drawback, since the input-output structure cannot be chosen arbitrarily. The second, and more severe drawback is that they are critically hampered by the so-called dissipation obstacle (Ortega, 2001). The dissipation obstacle dictates that no states can be stabilized that are subject to pervasive dissipation. Several methods have been developed to circumvent the dissipation obstacle, see e.g., (Ortega, 2001, 2003; Jeltsema, 2004; Ortega, 2008). However, in general, these methods rely on changing the actual output of the system and thus require a particular input-output structure, which is not always possible in practice.

These considerations call for a more general output feedback method. In this paper, we concentrate on the aforementioned second drawback and show that by removing the passivity constraint on the controller, i.e., by allowing for an active controller, the dissipation obstacle can simply be resolved while stability of the controller is established by using the Casimir relation between the plant and controller states. The design of output feedback controllers for non-collocated input-output systems will be treated elsewhere.

\section{CONTROL BY INTERCONNECTION AND CASIMIR FUNCTIONS}

In this section, we briefly review the control by interconnection (CbI) method applied to port-Hamiltonian ( $\mathrm{pH})$ plant systems of the form

$$
\begin{aligned}
& \dot{x}=[J(x)-R(x)] \frac{\partial H}{\partial x}(x)+G(x) u, \\
& y=G^{T}(x) \frac{\partial H}{\partial x}(x),
\end{aligned}
$$

where $x \in \mathbb{R}^{n}, u, y \in \mathbb{R}^{m}, J(x)$ an $n \times n$ matrix satisfying $J(x)=-J^{T}(x), R(x)$ an $n \times n$ matrix satisfying $R(x)=$ 
$R^{T}(x), G(x)$ an $n \times m$ matrix, and the Hamiltonian $H(x)$ represents the total stored energy. The matrix $J(x)$ is usually referred to as the interconnection structure, while $R(x)$ captures the resistive structure and is assumed to be positive semi-definite.

If the controller is also a $\mathrm{pH}$ system of the form

$$
\begin{aligned}
\dot{\xi} & =\left[J_{c}(\xi)-R_{c}(\xi)\right] \frac{\partial H_{c}}{\partial \xi}(\xi)+G_{c}(\xi) u_{c}, \\
y_{c} & =G_{c}^{T}(\xi) \frac{\partial H_{c}}{\partial \xi}(\xi)
\end{aligned}
$$

where $\xi \in \mathbb{R}^{n_{c}}, u_{c}, y_{c} \in \mathbb{R}^{m_{c}}, J_{c}(\xi)$ an $n_{c} \times n_{c}$ matrix satisfying $J_{c}(\xi)=-J_{c}^{T}(\xi), R_{c}(\xi)$ an $n_{c} \times n_{c}$ matrix satisfying $R_{c}(\xi)=R_{c}^{T}(\xi), G_{c}(\xi)$ an $n_{c} \times m_{c}$ matrix, and $H_{c}(\xi)$ represents the controller energy, then the interconnection of the plant system (1) with (2) via the standard (powerpreserving) feedback interconnection $u=-y_{c}, u_{c}=y$ (assuming $m=m_{c}$ ), yields the closed-loop system

$$
\begin{aligned}
& {\left[\begin{array}{c}
\dot{x} \\
\dot{\xi}
\end{array}\right]=\left[\begin{array}{cc}
J(x)-R(x) & -G(x) G_{c}^{T}(\xi) \\
G_{c}(\xi) G^{T}(x) & J_{c}(\xi)-R_{c}(\xi)
\end{array}\right]\left[\begin{array}{l}
\frac{\partial H}{\partial x}(x) \\
\frac{\partial H_{c}}{\partial \xi}(\xi)
\end{array}\right],} \\
& {\left[\begin{array}{c}
y \\
y_{c}
\end{array}\right]=\left[\begin{array}{cc}
G^{T}(x) & 0 \\
0 & G_{c}^{T}(\xi)
\end{array}\right]\left[\begin{array}{l}
\frac{\partial H}{\partial x}(x) \\
\frac{\partial H_{c}}{\partial \xi}(\xi)
\end{array}\right],}
\end{aligned}
$$

which is again a pH system.

The closed-loop system (3) is stabilized if the closed-loop Hamiltonian $H(x)+H_{c}(\xi)$ can be shaped such that it has a minimum at the desired equilibrium point $x^{*}$. The usual way to proceed is by restricting the motion of the closedloop system to the subspace

$$
\Omega=\left\{(x, \xi) \in \mathbb{R}^{n \times n_{c}} \mid C(x, \xi)=\kappa\right\},
$$

with $C(x, \xi)=\xi-S(x)$, where $S(x)$ is assumed to be a differentiable function and $\kappa \in \mathbb{R}$ is some constant, and such that the closed-loop Hamiltonian becomes $H(x)+$ $H_{c}(S(x)+\kappa)$. This is accomplished if, along the trajectories of (3), the functions $S(x)$ are such that

$$
\left.\dot{C}(x, \xi)\right|_{\Omega}=0 .
$$

The functions $C(x, \xi)$ are called Casimir functions and are independent of the Hamiltonian. We are thus looking for solutions $S(x)$ of the partial differential equations (PDE's)

$$
\left[-\frac{\partial^{T} S}{\partial x}(x) I_{n_{c}}\right]\left[\begin{array}{ll}
J(x)-R(x) & -G(x) G_{c}^{T}(\xi) \\
G_{c}(\xi) G^{T}(x) & J_{c}(\xi)-R_{c}(\xi)
\end{array}\right]=0,
$$

which, under the assumption that $R(x) \succeq 0$ and $R_{c}(\xi) \succeq$ 0 , are characterized by the following chain of equalities (Schaft, 2000):

$$
\begin{aligned}
\frac{\partial^{T} S}{\partial x}(x) J(x) \frac{\partial S}{\partial x}(x) & =J_{c}(\xi), \\
R(x) \frac{\partial S}{\partial x}(x) & =0, \\
R_{c}(\xi) & =0, \\
J(x) \frac{\partial S}{\partial x}(x) & =-G(x) G_{c}^{T}(\xi) .
\end{aligned}
$$

Unfortunately, the application of the $\mathrm{CbI}$ method is severely stymied by the condition (7), which, roughly speaking, dictates that the Casimir functions cannot depend on the coordinates that are subject to dissipation. This means that dissipation is admissible only on the coordinates of the closed-loop Hamiltonian that do not require shaping. For that reason, this condition is referred to as the dissipation obstacle (Ortega, 2001). However, the dissipation obstacle stems from the assumption that both the plant and controller dissipation structures satisfy $R(x) \succeq 0$ and $R_{c}(\xi) \succeq 0$. Although these properties are necessary to ensure that the plant and the controller are both passive systems, they are merely sufficient for passivity of the closed-loop system. In fact, the passivity assumption of the controller is unduly restrictive, as is illustrated in the following example.

\section{A MOTIVATING EXAMPLE}

Consider the RLC circuit shown in Fig. 1. Let $\phi$ denote the flux associated to the inductor and $q$ denote the charge associated to the capacitor, then the equations of motion are given by the $\mathrm{pH}$ description

$$
\begin{aligned}
{\left[\begin{array}{l}
\dot{\phi} \\
\dot{q}
\end{array}\right] } & =\left[\begin{array}{cc}
0 & -1 \\
1 & -1 / r
\end{array}\right]\left[\begin{array}{l}
\frac{\partial H}{\partial \phi}(\phi, q) \\
\frac{\partial H}{\partial q}(\phi, q)
\end{array}\right]+\left[\begin{array}{l}
1 \\
0
\end{array}\right] u, \\
y & =\left[\begin{array}{ll}
1 & 0
\end{array}\right]\left[\begin{array}{l}
\frac{\partial H}{\partial \phi}(\phi, q) \\
\frac{\partial H}{\partial q}(\phi, q)
\end{array}\right]
\end{aligned}
$$

where the Hamiltonian $H(\phi, q)=\frac{1}{2 L} \phi^{2}+\frac{1}{2 C} q^{2}$ represents the total stored energy.

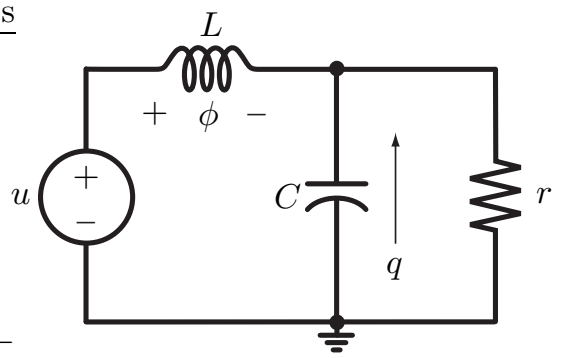

Fig. 1. RLC with pervasive dissipation.

This circuit was brought forward in (Ortega, 2001) as one of the simplest examples of a system that suffers from the dissipation obstacle. Indeed, since the equilibrium point equals $\left(\phi^{*}, q^{*}\right)=\left(L u^{*} / r, C u^{*}\right)$, which is non-zero for all $u^{*} \neq 0$, we need to shape the Hamiltonian in both coordinates, but condition (7) dictates that

$$
\frac{1}{r} \frac{\partial S}{\partial q}(\phi, q)=0 \text {. }
$$

Hence $S$ can only depend on the flux linkage $\phi$, instead of both $\phi$ and $q$ as required.

On the other hand, it is easily shown that the circuit is trivially stabilized by a feedforward control of the form $u=u^{*}$. Although this controller is not passive, it does 
allow for an energy shaping interpretation since by setting $u=u^{*}$ the circuit dynamics (10) become

$$
\begin{aligned}
{\left[\begin{array}{c}
\dot{\phi} \\
\dot{q}
\end{array}\right] } & =\left[\begin{array}{cc}
0 & -1 \\
1 & -1 / r
\end{array}\right]\left[\begin{array}{l}
\phi / L \\
q / C
\end{array}\right]+\left[\begin{array}{l}
1 \\
0
\end{array}\right] u^{*} \\
& =\left[\begin{array}{cc}
0 & -1 \\
1 & -1 / r
\end{array}\right]\left[\begin{array}{l}
\phi / L \\
q / C
\end{array}\right]+\left[\begin{array}{cc}
0 & -1 \\
1 & -1 / r
\end{array}\right]\left[\begin{array}{cc}
0 & -1 \\
1 & -1 / r
\end{array}\right]^{-1}\left[\begin{array}{l}
1 \\
0
\end{array}\right] u^{*} \\
& =\left[\begin{array}{cc}
0 & -1 \\
1 & -1 / r
\end{array}\right]\left[\begin{array}{c}
\phi / L-u^{*} / r \\
q / C-u^{*}
\end{array}\right]
\end{aligned}
$$

which can be associated to a shaped Hamiltonian of the form

$$
H_{d}(\phi, q)=\frac{1}{2 L}\left(\phi-L u^{*} / r\right)^{2}+\frac{1}{2 C}\left(q-C u^{*}\right)^{2}
$$

such that

$$
\left[\begin{array}{c}
\dot{\phi} \\
\dot{q}
\end{array}\right]=\left[\begin{array}{cc}
0 & -1 \\
1 & -1 / r
\end{array}\right]\left[\begin{array}{l}
\frac{\partial H_{d}}{\partial \phi}(\phi, q) \\
\frac{\partial H_{d}}{\partial q}(\phi, q)
\end{array}\right] .
$$

\section{BEYOND THE DISSIPATION OBSTACLE}

In the previous section, it is shown that the dissipation obstacle is a direct consequence of the passivity requirement on both the system and the controller. In this section, we show that by removing this constraint, the dissipation obstacle is easily resolved. The issue that remains then, is how the closed loop can be rendered stable. Using the Casimir relation between the controller state $\xi$ and the plant state $x$, we show that the static state feedback interpretation of the closed-loop system solves this issue in a straightforward manner.

Consider again the system of PDE's (5), or equivalently,

$$
\begin{aligned}
& \frac{\partial^{T} S}{\partial x}(x)[J(x)-R(x)]-G_{c}(\xi) G^{T}(x)=0, \\
& \frac{\partial^{T} S}{\partial x}(x) G(x) G_{c}^{T}(\xi)+\left[J_{c}(\xi)-R_{c}(\xi)\right]=0 .
\end{aligned}
$$

Substituting (12) into the transposed of (13) yields

$$
\frac{\partial^{T} S}{\partial x}(x)[J(x)-R(x)] \frac{\partial S}{\partial x}(x)-\left[J_{c}(\xi)+R_{c}(\xi)\right]=0
$$

which, after separation of the symmetric and skewsymmetric part, implies for the relationship between the controller and the plant dissipation and interconnection structure that

$$
\begin{aligned}
R_{c}(\xi) & =-\frac{\partial^{T} S}{\partial x}(x) R(x) \frac{\partial S}{\partial x}(x) \\
J_{c}(\xi) & =\frac{\partial^{T} S}{\partial x}(x) J(x) \frac{\partial S}{\partial x}(x),
\end{aligned}
$$

respectively. It is directly observed that (15) coincides with condition (6), whereas (14) coincides with (7) and (8) if, and only if, we only allow for dissipation structures satisfying $R(x) \succeq 0$ and $R_{c}(\xi) \succeq 0$. However, if we also allow for a non-positive controller dissipation structure, we can simply proceed from the closed-loop plant dynamics

$$
\dot{x}=[J(x)-R(x)] \frac{\partial H}{\partial x}(x)-G(x) G_{c}^{T}(\xi) \frac{\partial H_{c}}{\partial \xi}(\xi),
$$

which, by transposing (12), i.e.,

$$
G(x) G_{c}^{T}(\xi)=-[J(x)+R(x)] \frac{\partial S}{\partial x}(x),
$$

can be rewritten as

$$
\begin{aligned}
\dot{x}=[J(x) & -R(x)] \frac{\partial H}{\partial x}(x) \\
& +[J(x)+R(x)] \frac{\partial S}{\partial x}(x) \frac{\partial H_{c}}{\partial \xi}(\xi) .
\end{aligned}
$$

Hence, if $J(x)-R(x)$ is invertible, we can write

$$
\begin{aligned}
\dot{x}=[J(x)-R(x)]\left(\frac{\partial H}{\partial x}(x)+[J(x)-R(x)]^{-1}\right. \\
\left.\times[J(x)+R(x)] \frac{\partial S}{\partial x}(x) \frac{\partial H_{c}}{\partial S}(S(x)+\kappa)\right),
\end{aligned}
$$

which, after suitable choices of the controller Hamiltonian $H_{c}(S(x)+\kappa)$, may be interpreted as an energy shaping (ES) process such that the closed-loop plant dynamics take the form

$$
\dot{x}=[J(x)-R(x)] \frac{\partial H_{d}}{\partial x}(x)
$$

with

$$
\begin{aligned}
\frac{\partial H_{d}}{\partial x}(x) & =\frac{\partial H}{\partial x}(x)+[J(x)-R(x)]^{-1} \\
& \times[J(x)+R(x)] \frac{\partial S}{\partial x}(x) \frac{\partial H_{c}}{\partial S}(S(x)+\kappa),
\end{aligned}
$$

satisfying

$$
\frac{\partial^{2} H_{d}}{\partial x^{2}}(x)=\left(\frac{\partial^{2} H_{d}}{\partial x^{2}}(x)\right)^{T}, \text { for all } x \in \mathbb{R}^{n} .
$$

Furthermore, if $R(x) \succeq 0$, and

$$
\frac{\partial H_{d}}{\partial x}\left(x^{*}\right) \equiv 0, \quad \frac{\partial^{2} H_{d}}{\partial x^{2}}\left(x^{*}\right) \succ 0,
$$

it follows that $x^{*}$ is a stable equilibrium of (16).

In general, stability of (16) does not necessarily imply closed-loop stability. However, since $S: \mathbb{R}^{n} \rightarrow \mathbb{R}^{n_{c}}$ is differentiable by assumption (as is needed in (5)), it is continuous. By virtue of the well-known preservation of convergence under continuous mappings (Kolmogorov, 1999 ), as $x$ converges to $x^{*}$, the controller state $\xi$ converges to $\xi^{*}=S\left(x^{*}\right)$. Hence, stability of $x$ implies stability of $\xi$. This means that the controller does not need to have a positive semi-definite dissipation structure in order to be stable in the closed loop.

Although the method described above extends the traditional CbI method considerably, it is still hampered by the assumption that $J(x)-R(x)$ is invertible, and that (19) should be satisfied. Both these assumptions are needed for a pure ES interpretation of this CbI method, but are, in general, overly restrictive. However, using arguments similar to those of the static state feedback IDA-PBC method, the $\mathrm{CbI}$ method above can be extended to a dynamic output feedback IDA-PBC method. Indeed, starting from (16), we then have to look for matrices $J_{d}(x)=-J_{d}^{T}(x)$ and $R_{d}(x)=R_{d}^{T}(x)$, and a function $H_{d}: \mathbb{R}^{n} \rightarrow \mathbb{R}$ satisfying (19), such that

$$
\begin{aligned}
& \dot{x}=[J(x)-R(x)] \frac{\partial H}{\partial x}(x) \\
&+[J(x)+R(x)] \frac{\partial S}{\partial x}(x) \frac{\partial H_{c}}{\partial S}(S(x)+\kappa) \\
& \equiv\left[J_{d}(x)-R_{d}(x)\right] \frac{\partial H_{d}}{\partial x}(x) .
\end{aligned}
$$


This result effectively generalizes both the standard passive ES Casimir-based control method as presented in Section 2, and the extended ES Casimir-based control method of (16)-(18), to a dynamic output feedback IDA-PBC strategy. Summarizing, we have the following proposition.

Proposition. Consider the interconnection of the plant (1) with the controller (2), assume that (5) holds, and that the closed-loop plant dynamics satisfy (21) (resp. (18)). Then, if the closed-loop plant Hamiltonian $H_{d}(x)$ satisfies $(20)$ and $R_{d}(x) \succeq 0$ (resp. $R(x) \succeq 0$ ), the equilibrium point $\left(x^{*}, S\left(x^{*}\right)\right)$ of the overall system (3) is stable.

\section{A MOTIVATING EXAMPLE (CONT'D)}

Let us return to the RLC circuit of Fig. 1. In Section 3, we have seen that the circuit suffers from the dissipation obstacle. However, suppose we interconnect, again via standard feedback, the circuit (10) with a pH controller of the form

$$
\begin{aligned}
\dot{\xi} & =-R_{c} \frac{\partial H_{c}}{\partial \xi}(\xi)+G_{c} u_{c}, \\
y_{c} & =G_{c} \frac{\partial H_{c}}{\partial \xi}(\xi) .
\end{aligned}
$$

Then, proceeding from (5), we find

$$
\left.\begin{array}{rl}
\frac{\partial S}{\partial q}(\phi, q)-G_{c} & =0 \\
\frac{\partial S}{\partial \phi}(\phi, q)+\frac{1}{r} \frac{\partial S}{\partial q}(\phi, q) & =0 \\
\frac{\partial S}{\partial \phi}(\phi, q) G_{c}-R_{c} & =0
\end{array}\right\} \Leftrightarrow S(\phi, q)=G_{c}(q-\phi / r)
$$

and $R_{c}=-\frac{1}{r} G_{c}^{2}$, which is non-positive for all $r<\infty$ and $G_{c} \neq 0$.

\subsection{Recovering the Feedforward Controller}

Now, setting the controller Hamiltonian $H_{c}(\xi)=\xi$, with $\xi=S(\phi, q)$, the dynamics of the controlled circuit becomes

$$
\begin{aligned}
{\left[\begin{array}{c}
\dot{\phi} \\
\dot{q}
\end{array}\right]=} & {\left[\begin{array}{cc}
0 & -1 \\
1 & -1 / r
\end{array}\right]\left[\begin{array}{l}
\frac{\partial H}{\partial \phi}(\phi, q) \\
\frac{\partial H}{\partial q}(\phi, q)
\end{array}\right]+\left[\begin{array}{l}
1 \\
0
\end{array}\right]\left(-y_{c}\right) } \\
= & {\left[\begin{array}{cc}
0 & -1 \\
1 & -1 / r
\end{array}\right]\left[\begin{array}{l}
\frac{\partial H}{\partial \phi}(\phi, q) \\
\frac{\partial H}{\partial q}(\phi, q)
\end{array}\right] } \\
& -\left[\begin{array}{ll}
0 & -1 \\
1 & -1 / r
\end{array}\right]\left[\begin{array}{ll}
0 & -1 \\
1 & -1 / r
\end{array}\right]^{-1}\left[\begin{array}{l}
1 \\
0
\end{array}\right] G_{c} \frac{\partial H_{c}}{\partial \xi}(\xi) \\
= & {\left[\begin{array}{ll}
0 & -1 \\
1 & -1 / r
\end{array}\right]\left[\begin{array}{l}
\frac{\partial H}{\partial \phi}(\phi, q)+\frac{G_{c}}{r} \frac{\partial H_{c}}{\partial \xi}(\xi) \\
\frac{\partial H}{\partial q}(\phi, q)+G_{c} \frac{\partial H_{c}}{\partial \xi}(\xi)
\end{array}\right] . }
\end{aligned}
$$

Since $\frac{\partial H_{c}}{\partial \xi}(\xi)=1$, the stabilization problem is trivially solved by setting $G_{c}=-u^{*}$ such that (23) is equivalent to

$$
\left[\begin{array}{c}
\dot{\phi} \\
\dot{q}
\end{array}\right]=\left[\begin{array}{cc}
0 & -1 \\
1 & -1 / r
\end{array}\right]\left[\begin{array}{l}
\frac{\partial H_{d}}{\partial \phi}(\phi, q) \\
\frac{\partial H_{d}}{\partial q}(\phi, q)
\end{array}\right]
$$

with shaped Hamiltonian

$$
H_{d}(\phi, q)=\frac{1}{2 L}\left(\phi-L u^{*} / r\right)^{2}+\frac{1}{2 C}\left(q-C u^{*}\right)^{2} .
$$

At this point it is important to emphasize that, although the circuit is stabilized by a constant control, the underlying controller dynamics (22) take the form

$$
\begin{aligned}
\dot{\xi} & =\frac{1}{r}\left(u^{*}\right)^{2}-u^{*} u_{c}, \\
y_{c} & =-u^{*} .
\end{aligned}
$$

Despite the fact that $R_{c}<0$, for all $u^{*} \neq 0$, the controller dynamics is asymptotically stable since from (24) we may deduce that $\phi \rightarrow L u^{*} / r$ and $q \rightarrow C u^{*}$, as $t \rightarrow \infty$, which, since $\xi=S(\phi, q)$, implies that the controller state converges to $\xi^{*}=-u^{*}\left(q^{*}-\phi^{*} / r\right)$. Also note that $u_{c} \rightarrow$ $u^{*} / r$ implies $\dot{\xi} \rightarrow 0$.

The Casimir approach thus leads us naturally to the feedforward controller that is known to stabilize the system, and in spite of its feedforward character, the controller dynamics are instrumental for the construction of the controller.

\subsection{Output Feedback Control}

Let us next exploit the freedom in choosing $H_{c}(\xi)$ as

$$
H_{c}(\xi)=\frac{1}{2} a_{1} \xi^{2}+a_{2} \xi
$$

with $\xi=S(\phi, q)$ and $a_{i} \in \mathbb{R}$, for $i=1,2$, some constants to be defined. With this choice, however, it is not possible to interpret the control action as an ES process (18) since

$$
\left[\begin{array}{l}
\frac{\partial H}{\partial \phi}(\phi, q) \\
\frac{\partial H}{\partial q}(\phi, q)
\end{array}\right]+\left[\begin{array}{cc}
1 & 2 / r \\
0 & 1
\end{array}\right]\left[\begin{array}{l}
\frac{\partial S}{\partial \phi}(\phi, q) \\
\frac{\partial S}{\partial q}(\phi, q)
\end{array}\right] \frac{\partial H_{c}}{\partial S}(S(\phi, q))
$$

is not a gradient vector field to which we can associate a Hamiltonian $H_{d}(\phi, q)$ satisfying (19). On the other hand, the closed-loop dynamics of the plant take the form

$$
\left[\begin{array}{c}
\dot{\phi} \\
\dot{q}
\end{array}\right]=\left[\begin{array}{cc}
a_{1} G_{c} L / r & -\left(1+a_{1} G_{c} C\right) \\
1 & -1 / r
\end{array}\right]\left[\begin{array}{l}
\phi / L \\
q / C
\end{array}\right]+\left[\begin{array}{c}
-G_{c} a_{2} \\
0
\end{array}\right],
$$

which can be rewritten in $\mathrm{pH}$ form as

$$
\begin{aligned}
{\left[\begin{array}{c}
\dot{\phi} \\
\dot{q}
\end{array}\right]=} & (\underbrace{\left[\begin{array}{cc}
0 & -1-a_{1} G_{c} C / 2 \\
1+a_{1} G_{c} C / 2 & 0
\end{array}\right]}_{J_{d}} \\
& -\underbrace{\left[\begin{array}{cc}
-a_{1} G_{c} L / r & a_{1} G_{c} C / 2 \\
a_{1} G_{c} C / 2 & 1 / r
\end{array}\right]}_{R_{d}})\left[\begin{array}{l}
\frac{\partial H_{d}}{\partial \phi}(\phi, q) \\
\frac{\partial H_{d}}{\partial q}(\phi, q)
\end{array}\right],
\end{aligned}
$$

where

$$
H_{d}(\phi, q)=\frac{1}{2 L}(\phi-L \alpha / r)^{2}+\frac{1}{2 C}(q-C \alpha)^{2}
$$

with $\alpha=-r^{2} a_{2} G_{c}\left(r^{2}+a_{1} C G_{c} r^{2}-a_{1} G_{c} L\right)^{-1}$. 
Note that the closed-loop plant dynamics is stable if the controller parameters $a_{1}$ and $G_{c}$ are selected such that $R_{d} \succeq 0$. The desired equilibrium point is determined by an appropriate selection of $a_{2}$.

\section{FINAL REMARKS AND OUTLOOK}

In this paper, an extension of the Casimir-based controlby-interconnection method is presented that resolves the so-called dissipation obstacle. This result is based on removing the passivity constraint on the controller and using the Casimir relation between the plant and controller states to guarantee stability of the closed loop.

Using a simple RLC circuit example with pervasive dissipation, two intriguing controller synthesis solutions are presented. First, it is shown that this dynamic outputfeedback based design methodology is able to produce a feedforward controller, while the second solution shows how the dynamics of the controller are instrumental in shaping the energy in the coordinates that are not used for feedback.

Although the results presented in this paper only emphasize the possibility of an active controller, future work will include conditions under which also active plants are allowed, as well as the consideration of $\mathrm{pH}$ systems with direct feedthrough and output feedback for non-collocated input-output channels.

\section{REFERENCES}

V. Duindam, A. Macchelli, S. Stramigioli and H. Bruyninckx, "Modeling and Control of Complex Physical Systems", Springer, 2009

A.N. Kolmogorov, and S.V. Fomin, Elements of the Theory of Functions and Functional Analysis, Dover Publications Inc., Mineola, NY, 1999.

R. Ortega, D. Jeltsema and J. M. A. Scherpen, "Power shaping: a new paradigm for stabilization of nonlinear RLC circuits", IEEE Transactions on Automatic Control, vol. 48, pp. 1762-1767, 2003.

D. Jeltsema, R. Ortega and J.M.A. Scherpen, "An energybalancing perspective of interconnection and damping assignment control of nonlinear systems", Automatica, Vol. 40, No. 9, September 2004, pp. 1643-1646.

R. Ortega, A.J. van der Schaft, F. Castanos, and A. Astolfi, "Control by interconnection and standard passivity-based control of port-Hamiltonian systems", IEEE Transactions on Automatic Control, vol. 53, pp. 2527-2542, 2008.

R. Ortega, A.J. van der Schaft, I. Mareels, and B.M. Maschke, "Putting energy back in control", Control Systems Magazine, vol. 21, pp. 18-33, 2001.

A.J. van der Schaft, $L_{2}-$ Gain and Passivity Techniques in Nonlinear Control, Springer-Verlag, London, 2000. 